\author{
Max Aebi \\ Robert Gunzburg \\ Hans-Joachim Wilke
}

\section{Manuscript submission and processing: the new electronic pathway}

As of January 2004, Springer-Verlag will introduce an online system for manuscript submission and editorial processing. With ManuscriptCentral (MC), all processing of European Spine Journal manuscripts, from submittal to acceptance for publication, will be exclusively electronic and online. The author's initial submittal of a manuscript will be online (rather than via a mailed paper copy). The editor, associate editors, and reviewers will all retrieve the manuscript online, the author will receive reviewer comments and the publication decision by e-mail and submit a revised manuscript online, and the final accepted manuscript will be forwarded to the publisher online. Everyone will have access to the

Published online: 25 November 2003 (C) Springer-Verlag 2003

\author{
M. Aebi (®) \\ University of Bern, \\ Institute for Evaluative Research \\ in Orthopaedic Surgery, \\ Murtenstrasse 35, 3008 Bern, Switzerland \\ e-mail: max.aebi@MEMcenter.unibe.ch \\ R. Gunzburg \\ Eeuwfeestkliniek, \\ Harmoniestraat 68, \\ 2018 Antwerp, Belgium \\ H.-J. Wilke \\ Institute for Trauma Surgery Research \\ and Biomechanics, University of Ulm, \\ Helmholtzstrasse 14, \\ 89081 Ulm, Germany
}

same online system. The publisher of the European Spine Journal has provided two online systems for some time now - online proof checking and online publication of each manuscript prior to paper publication. The new steps that we are announcing here will make the processing of a European Spine Journal manuscript, from submittal to publication, completely electronic.

These new arrangements should provide several advantages: mailing and courier costs can be reduced for authors and European Spine Journal staff because most of the transactions will be done online. Authors need not make and send multiple copies of their manuscripts, but can prepare their initial manuscript in the exact final electronic format required for publication. The length of time between the manuscript submittal and the publication decision will be shorter because manuscript transactions will not only be transported nearly instantaneously, but also carefully tracked by the European Spine Journal editorial office. This office will prompt all editors and reviewers to keep to the promised completion schedules for each review step of each manuscript.

Logging into MC for electronic manuscript submission is simple. Online, connect to: http://esj.manuscriptcentral.com. The first time you do this, please check for an existing account by clicking the "check for existing account" button. If an account exists, you will then receive an automatic e-mail containing your user ID and password. If no account exists, please click on "create a new account" and follow the instructions provided on the screen - a new user ID and password will then be e-mailed to you.

Once you have an account, simply type in your user ID and password, and MC will open for you. Complete instructions and help regarding online submittal of a manuscript can be found in MC. After logging in, authors may submit a new manuscript or track a previously submitted manuscript, and reviewers may open and download manuscripts for review. Users' access to information in MC depends on their roles: authors may only view some information regarding their own manuscripts; reviewers may access the manuscripts and review forms; associate editors may additionally access reviewer comments; and the executive editor may view all information for all manuscripts. Paper manuscript submittal will be accepted by European Spine Journal only if it is impossible for the author to submit a manuscript online. If for some reason the authors cannot access MC, they are asked to send electronic text files and graphics so that all the documents may be submitted online by the administrative office instead of the author. 